\title{
Ultrabroad-Band Direct Digital Refractive Index Imaging Based on Suspended Graphene Plasmon Nanocavities
}

\author{
Xiaofei Xiao,* Stefan A. Maier, and Vincenzo Giannini
}

Cite This: ACS Appl. Nano Mater. 2021, 4, 1635-1642

Read Online

ABSTRACT: Mid-infrared spectroscopy is essential for chemical identification and compositional analysis because of the existence of characteristic molecular absorption fingerprints. However, it is very challenging to determine the refractive index of an analyte at low concentrations using current photonic systems in a broad midinfrared spectral range. We propose an imaging-based nanophotonic technique for refractive index determination. The technique is based on deep subwavelength graphene plasmon nanocavities and allows for the retrieval of molecular concentration. This method features a two-dimensional array of suspended graphene plasmon nanocavities, in which the extremely high field enhancement and extraordinary compression of graphene plasmons can be realized simultaneously by combining shallow and deep cavities. This enables resonant unit cells to be read out in the spatial absorption pattern of the array at multiple spectral points, and the resulting information is then

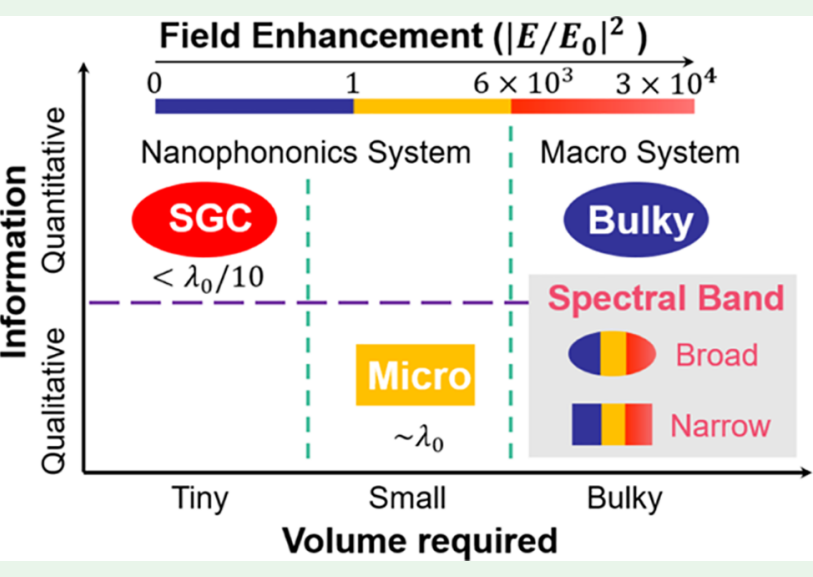
translated into the refractive index of the analytes. The proposed technique gives complementary information compared with the current nanophotonic techniques based on molecular absorption, including the ability of the refractive index measurement, the ultra-broadband measurable spectral range, and the small volume of the analyte required, thereby pushing the potential for refractometric sensing technologies into the infrared frequencies.

KEYWORDS: graphene plasmonics, suspended 2D materials, surface phonon polaritons, sensing, metamaterials

\section{INTRODUCTION}

The mid-infrared (MIR) spectrum is known for the presence of characteristic molecular absorption fingerprints originating from the intrinsic vibrational modes of chemical bonds, and thus, the MIR spectrum is critical for chemical identification and structural characterization. ${ }^{1,2}$ Conventionally, spectral analysis is achieved using macro systems, such as Fouriertransform infrared (FTIR) spectrometers, which measure the transmittance or emission spectrum of the analyte using gratings. ${ }^{3}$ The analyte can be characterized based on either the retrieved refractive index (Figure 1a) or the existence of the characteristic molecular absorption fingerprints. However, this bulk approach usually requires complex and expensive equipment, such as FTIR spectrometers, and suffers from low sensitivity when detecting signals from small volumes of samples, because of the mismatch between MIR wavelengths $(\sim \mu \mathrm{m})$ and the dimensions of molecules $(\sim \mathrm{nm})$.

To tackle this problem, nanophotonic systems with strong near-field enhancement of subwavelength resonators have been explored in MIR optical sensing. Recent sensor-on-chip approaches based on the surface-enhanced infrared absorption (SEIRA) have been realized based on various nanophotonic platforms, $^{1,4-9}$ which potentially allow for a simplified and inexpensive sensor design that is well suited to miniaturization. However, the achieved performance is still far from ideal. Most platforms are based on near-field enhancement of subwavelength resonators; however, the tuning band range of the resonance frequency in current platforms only covers a small spectral region. Furthermore, although plasmonic or alldielectric resonators are used, the field enhancement (IE/ $\left.E_{0}\right|^{2}$ ) is constrained to (usually) less than $10^{3}$ and field compression is limited to a scale around $\lambda_{0} / 10$ (where $\lambda_{0}$ is the free-space wavelength). ${ }^{10-13}$ This diminishes the sensitivity when looking at nanometer-scale samples at mid-IR wavelengths. In fact, most current sensor-on-chip approaches are based on the detection of characteristic molecular absorption. 6 Thus, they can be used for differentiating types of molecules; however, using these techniques, it is challenging to obtain a quantitative characterization of properties of the

Received: November 20, 2020

Accepted: January 25, 2021

Published: February 8, 2021 


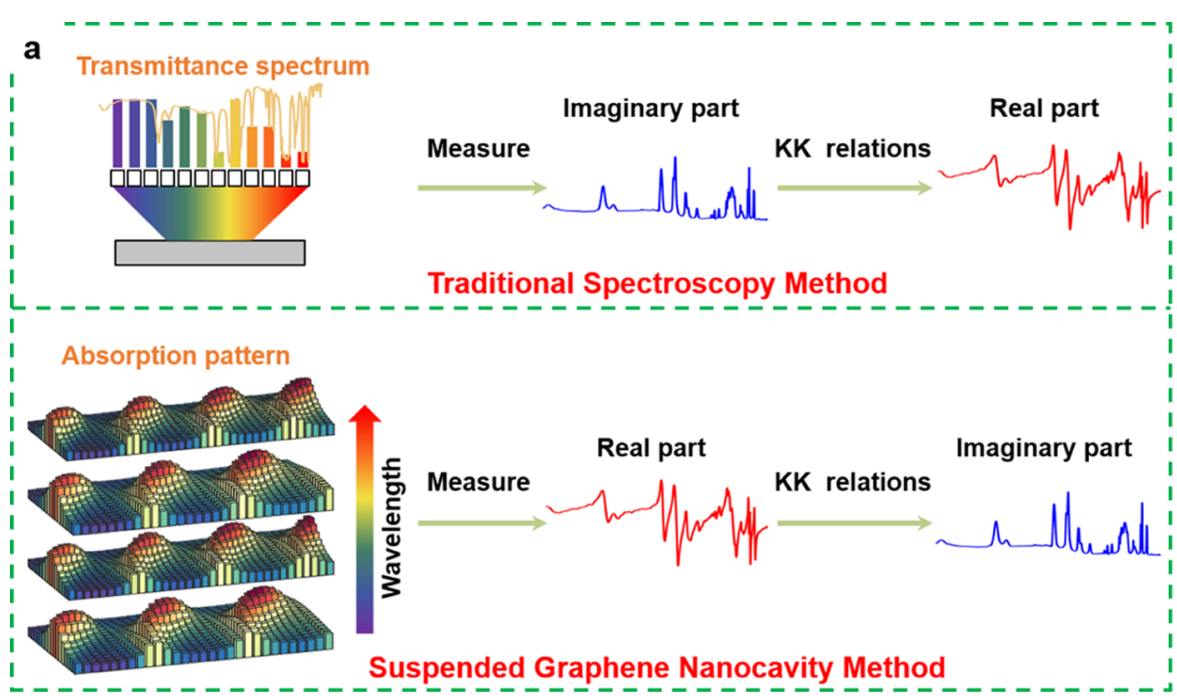

Figure 1. Schematic illustration of refractometric approaches. (a) Different operating mechanisms that enable retrieving the complex refractive index of an analyte. Within the traditional bulk method, using a spectrometer (top path), the transmittance spectrum is measured. Based on BeerLambert's relation, the imaginary part of the refractive index can be calculated. An alternative approach (bottom path), the proposed suspended graphene nanocavity method, is to measure the real part of the refractive index, which can be extracted by measuring the resonance pattern of a sensor array. KK relations are short for Kramers-Kronig relations. (b) Schematic illustration of the proposed technique based on suspended graphene nanocavities.

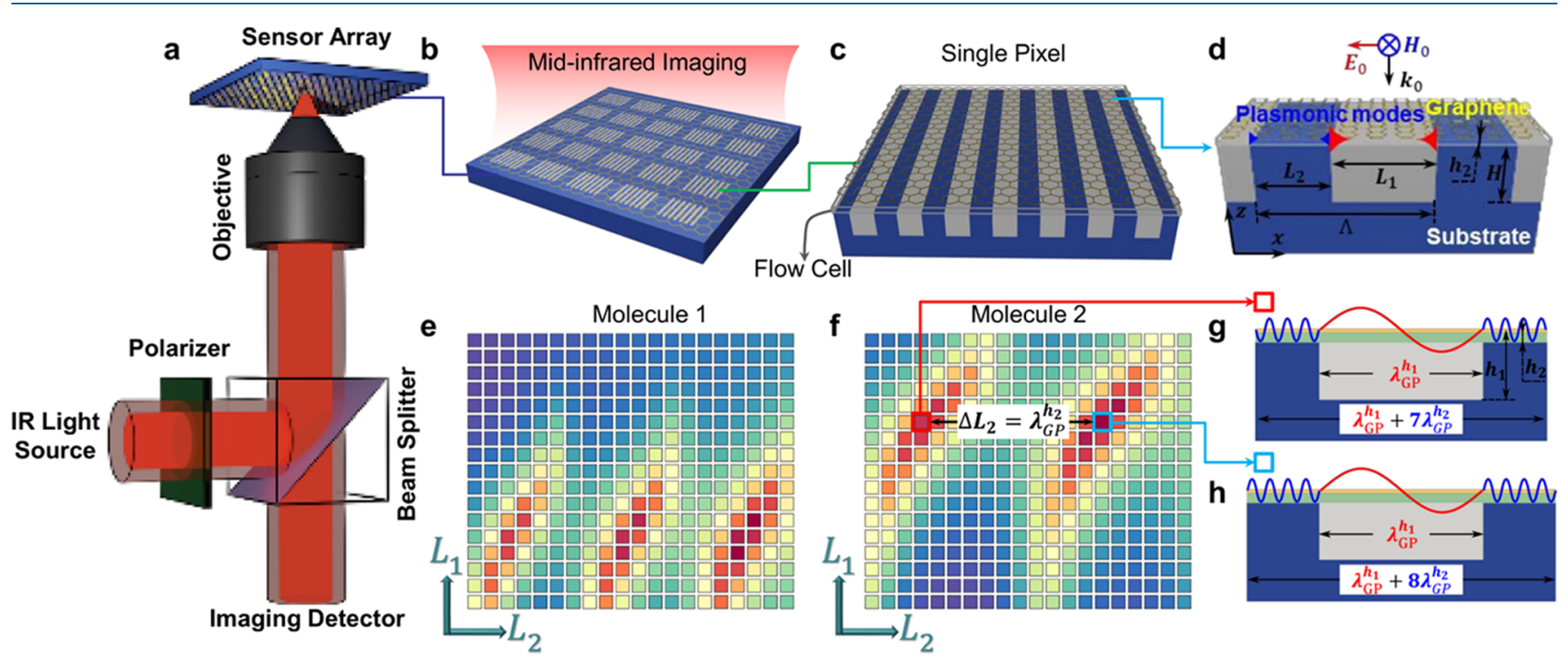

Figure 2. Molecular refractive index detection with a digital sensor using suspended graphene plasmon nanocavities. (a) Schematic illustration of the imaging-based MIR microscopy system. The monochromatic light generated by the light source focuses on a digital sensor after passing through a linear polarizer, a beam splitter, and an objective. The incidence is a transverse magnetic (TM) polarized light in terms of the grating surface and normal to the surface of the sample. The reflected light is collected and imaged by an infrared camera. (b) Chessboard sensor is composed of a two-dimensional array of suspended graphene plasmon nanocavities with resonances that are highly sensitive to the refractive index of the analyte. (c) Each building block is composed of a graphene monolayer deposited on a metallic grating. A flow cell is used to circulate liquid samples and support the graphene layer. (d) Schematic of grating-assisted suspended graphene plasmon nanocavities. The incidence is TM polarized in air, where $\Lambda, L_{1}, L_{2}$, and $H$ are the period, trench length, ridge length, height of the grating, and $h_{2}$ is the gap thickness. Thus, we have $h_{1}=h_{2}+H$. The gap and the trench can be filled with dielectric media. (e-f) Captured absorption patterns for two different molecules. In this work, we choose $L_{1}$ and $L_{2}$ as two variables of the array in the horizontal and vertical directions. Note that any one pair of the parameters (such as $L_{1}, L_{2}, h_{2}, H$, and $E_{\mathrm{F}}$ ) of the graphene plasmon nanocavity system could be used as the varying parameters of the array. The material in the flow cell has refractive index $n$. $(\mathrm{g}-\mathrm{h})$ Sketches of two resonance modes for two structures with different ridge lengths.

analyte such as the refractive index. This is because of the low signal-to-noise ratio originating from the relatively small imaginary part of the refractive indices of molecules and the small volume of the analyte used in these approaches.

Within a Fabry-Pérot cavity, the real part of the refractive index of the cavity materials determines the occurrence of the resonance, which is visible in the absorption of the system.
Therefore, the visibility of the resonance can be used to measure the refractive index of the material in the cavity. Such a technique has been realized in bulk systems. ${ }^{14,15}$ However, it is difficult to realize this in a deep subwavelength system because of the limited field compression and/or the large loss. Graphene plasmons ${ }^{16-19}$ (GPs) can overcome this limitation by exploiting their extremely short wavelength, strong field 

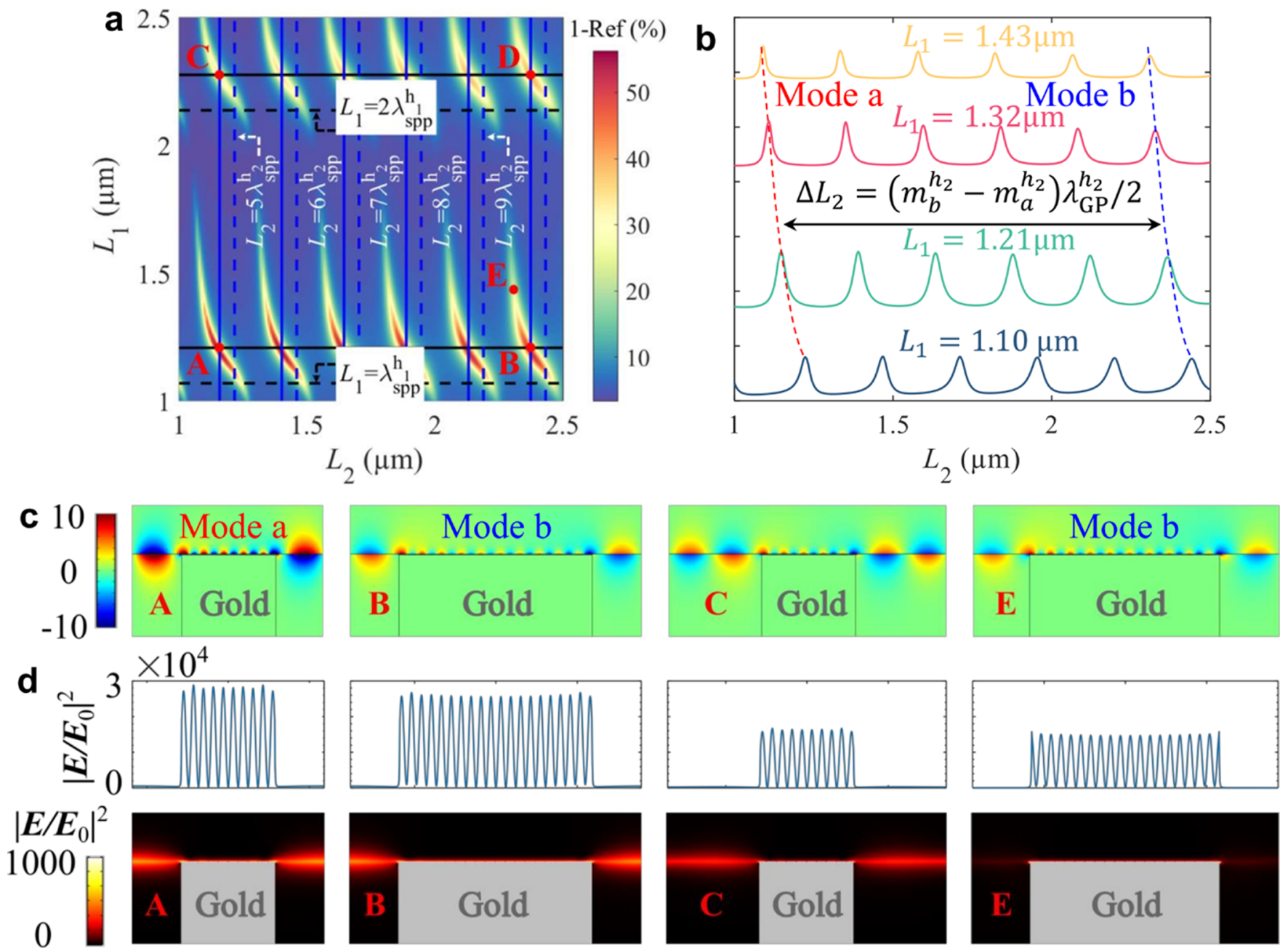

Figure 3. Resonance generation mechanisms in suspended graphene plasmon nanocavities. (a) Absorption of the system (1 - Ref, where Ref denotes the reflection) for the gold grating plus graphene structure versus the trench length $\left(L_{1}\right)$ and the ridge length $\left(L_{2}\right)$, when the gap thickness $\left(h_{2}\right)$ is $5 \mathrm{~nm}$ (normal incidence $\lambda_{0}=12 \mu \mathrm{m}$ ), where the trench and the gap are filled with air, and $E_{\mathrm{F}}=0.64 \mathrm{eV}$. The colored map is obtained from numerical simulations and the straight lines are from the proposed model. The dashed lines indicate a fit to a Fabry-Pérot model for a phase shift of 0 . The black (blue) solid lines indicate a fit to a Fabry-Pérot model for a phase shift of $-0.27 \pi(0.54 \pi)$. (b) Line cuts of the absorption in (a) for four trench lengths $\left(L_{1}\right)$ indicated by the labels. The orders for mode a and mode $\mathrm{b}$ are $m_{\mathrm{a}}^{h_{2}}=10$ and $m_{\mathrm{b}}^{h_{2}}=20$, respectively. (c) Component $E_{z}$ of the electric field is shown for the selected points marked by A, B, C, and E in (a). The corresponding parameters are as follows: A, $L_{1}=1.210 \mu \mathrm{m}$ and $L_{2}=1.150 \mu \mathrm{m} ; \mathrm{B}, L_{1}=1.210 \mu \mathrm{m}$ and $L_{2}=2.365 \mu \mathrm{m} ; \mathrm{C}, L_{1}=2.280 \mu \mathrm{m}$ and $L_{2}=1.150 \mu \mathrm{m} ; \mathrm{E}, L_{1}=1.450 \mu \mathrm{m}$ and $L_{2}=2.310 \mu \mathrm{m}$. The electric field is normalized to the incident field amplitude $E_{0}$. (d) Electric near-field with high-intensity enhancement $\left(\left|E / E_{0}\right|^{2}\right)$ is strongly compressed in the cavity above the ridge. Here, $\lambda_{0} / h_{2}=2400$. Distribution of the intensity along a line positioned in the middle of the gap is given as well.

confinement, and large field enhancement. Furthermore, GPs have a frequency range covering the mid- and far-IR and terahertz bands ${ }^{20,21}$ and can be dynamically tuned by changing the Fermi energy via an external gate voltage. ${ }^{22-24}$ This strong tunability combined with a broad spectral range makes GPs highly promising for various vital applications such as modulators $^{20}$ and photodetectors. ${ }^{25-29}$ In the last decade, many tunable sensors, ${ }^{30,31}$ including mid-infrared sensors, ${ }^{32-35}$ based on graphene have been proposed and investigated, indicating that graphene could offer a great opportunity to significantly improve the performance of devices used for refractometric detection in the infrared band.

In this work, we report on a broadband tunable sensing technique based on suspended graphene plasmon nanocavities (Figures $1 \mathrm{~b}$ and 2) and demonstrate its capability for enhancing light-matter interactions, measuring the real part of the refractive index of the analyte and characterizing both types and concentrations of the various molecules. Our design exploits the standing wave properties of plasmonic resonance in suspended graphene nanocavities, which is driven by two horizontal Fabry-Pérot cavities and one vertical Fabry-Pérot cavity (Figure 2c,d). In particular, we implement a twodimensional array of suspended graphene plasmon nanocavities, which consist of a continuous graphene monolayer on top of a metallic grating with a gap. The GPs are excited due to the scattering from the edges of the cavities, and then, they form two horizontal cavities in the gap and in the trench (Figure $2 \mathrm{~g}, \mathrm{~h}$ ). The resonance is highly sensitive to the real part of the refractive index of the analyte. Therefore, this configuration allows us to establish a unique relation between the real part of the refractive index of the analyte and the spatial absorption pattern (Figures $1 \mathrm{a}$ and $2 \mathrm{e}, \mathrm{f}$ ). By translating these spatial absorption maps for different molecules into their refractive indices, we demonstrate that this technique is suitable for refractive index measurement, chemical identification, and concentration analysis. The proposed technique 
offers several advantages in molecular sensing over traditional nanophotonic systems. These include the ability to measure refractive indices, the ultra-broadband measurable spectral range, the tiny volume of the analyte required, and the deep subwavelength dimensions of the elements.

\section{RESULTS AND DISCUSSION}

Plasmonic waves in graphene have attracted numerous investigations because of their extreme field confinement. However, the efficient excitation of such plasmonic waves is still very challenging because there is a large momentum mismatch between the incident light and GPs. In this work, the GPs are excited efficiently and the extremely high field enhancement and extraordinary compression of GPs occur simultaneously, thanks to the combination of a shallow cavity and a deep cavity in the same configuration: the shallow one is above the ridge with length $L_{2}$ and height $h_{2}$, and the deep one is in the trench with length $L_{1}$ and height $h_{1}$ (Figure 2d). In each cavity, the scattering by sharp edges is used to generate a broad spectrum of wavevectors to compensate for the momentum mismatch. The forward and backward launched plasmonic waves constructively interfere in the cavities to form standing waves (Figure $2 \mathrm{~g}, \mathrm{~h}$ ). Thus, when the cavity length $L_{j}$ satisfies the Fabry-Pérot equation (where $j=1,2$ represents the cavities in the trench and above the ridge, respectively), the GPs may be excited. A Fabry-Pérot equation reads

$$
\delta \phi_{j}+\mathcal{R}\left\{k_{\mathrm{GP}}^{h_{j}}\left(\lambda_{0}\right)\right\} L_{j}=m \pi, m=0,1,2,3 \ldots, j=1 \text { and } 2
$$

where $\delta \phi_{j}$ is the phase shift, $k_{\mathrm{GP}}^{h_{j}}$ denotes the wavenumber of the GPs, and the integer $m$ denotes the resonance mode order. The cavity height-dependent dispersion relation of GPs derived in ref 36 is used in this work. The real part of the wavenumber of GPs determines the effective plasmon wavelength, $\lambda_{\mathrm{GP}}^{h}=2 \pi / \mathcal{R}\left\{k_{\mathrm{GP}}^{h}\right\}$, where $h$ denotes the cavity height and could be $h_{1}$ or $h_{2}$ in this work. To study the properties of GPs, the effective refractive index (ERI) of the $\mathrm{GP}, N_{\mathrm{eff}}^{h}=k_{\mathrm{GP}}^{h} / k_{0}$ (where $k_{0}$ is the wavenumber in free space), is introduced. Taking a phase shift of 0 , we get $L_{j}=m \lambda_{\mathrm{GPj}}^{h} / 2$, which we find to be a good approximation for our results. Figure $2 \mathrm{~g}$,h shows the sketches of the standing waves of GP resonances supported in this system. While those waves propagate in the graphene layer, the optical energy is dissipated through Ohmic losses.

Based on the Fabry-Perot equation and the dispersion relation of GPs, we can establish a theory for the fundamental building block of our sensor, as shown in Figure 2c,d. The system is composed of a graphene monolayer deposited on a gold grating. We assume that there is a small gap (with a thickness of $h_{2}$ ) between the two. The gap and the trench are filled with the analyte and dielectric materials, respectively. Without loss of generality, we shall take the dielectric in the trench to be air. More realistic configurations will be discussed later. To investigate our system, we begin by numerically calculating the absorption of the system as a function of trench length $\left(L_{1}\right)$ and ridge length $\left(L_{2}\right)$ for a fixed gap thickness $h_{2}=$ $5 \mathrm{~nm}$ and a fixed incident wavelength $\lambda_{0}=12 \mu \mathrm{m}$. The trench high $(H)$ is $2 \mu \mathrm{m}$, unless otherwise specified. The Fermi energy of the graphene layer $\left(E_{F}\right)$ is $0.64 \mathrm{eV}$. According to the cavity height-dependent dispersion of GPs, ${ }^{36}$ the corresponding ERIs $\left(N_{\text {eff }}^{h}\right)$ are $49.3015+0.3239 i$ and $11.2314+0.1315 i$ with $h=5$ $\mathrm{nm}$ and $h>1 \mu \mathrm{m}$, respectively. The absorption of the system versus the trench length and the ridge length is shown in Figure 3a. The strong absorption peaks are because of the excitation of the GPs, which can be confirmed by near-field plots in Figure 3c.

In earlier works, ${ }^{36,37}$ the graphene monolayer and the grating were in physical contact with one another. However, because of the existence of the small gap in this study, there exists a very strong field in the parts of the graphene which are located above the ridge of the gold grating. Consequently, there is very strong absorption in these locations. In Figure 3a, the local maximum values of the graphene absorption correspond to the excitation of standing waves in both cavities. The predictions of those local peak positions are also given using the proposed theory, as the crossing points of the black dashed lines (for the cavity in the trench) and the blue dashed lines (for the cavity above the ridge) are shown in Figure 3a. Although the reasonable agreement between the predictions and the numerical results confirms the validity of the proposed model, there is an apparent shift between the predictions and the real local peak positions. This discrepancy is because of the coupling between the graphene plasmonic modes and the dipole mode of the metallic structure, which can be observed clearly in the near-field plots, as shown in Figure 3c. Therefore, we need to modify the Fabry-Pérot model by optimizing the phase shift $\delta \phi_{j}$ to a value different from 0 to compensate for the phase difference, as the figure caption shows. It should be noted that the phase shifts $\delta \phi_{j}$ strongly depend on the frequency of interest, the gap thickness, the permittivities of the materials in the system, and the properties of the graphene layer. Using the modified phase shifts, we demonstrate excellent predictions as shown by the crossing points between the black solid lines and the blue solid lines in Figure $3 \mathrm{a}$.

Another interesting feature is that strong absorption persists when the conditions for establishing standing waves in those two cavities are not fully satisfied individually, as confirmed by the near-field plot in Figure $3 \mathrm{c}$ for the point marked as point $\mathrm{E}$ in Figure 3a. The absorption is the strongest when the FabryPérot resonance is fully satisfied in each cavity. It is worth noting that, if we choose several trench lengths, as shown in Figure $3 b$, the difference in the position of the adjacent peaks equals the effective wavelength of GPs in the shallow cavities $\left(\lambda_{\mathrm{GP}}^{h_{2}}\right)$, which can be used to retrieve the refractive index of the material in the gap.

An appealing characteristic of suspended graphene nanocavity systems is that the electric field with extreme high intensity can be strongly compressed inside the gap above the ridge. Previous work ${ }^{36}$ has shown that the electromagnetic field can be trapped in cavities with compression factor around 400 or intensity enhancement around 4000. However, these two phenomena did not appear in the same system. In this work, we achieve both the extremely high enhancement and the extraordinary compression in the same system, as shown in Figure $3 \mathrm{~d}$. This is because (i) the tiny gap above the ridge makes the extraordinary compression possible, and (ii) the optimized trench height could significantly enhance the conversion efficiency using a horizontal Fabry-Pérot cavity. Higher enhancement and even more compression could be achieved by further optimizing the parameters of the system.

So far, we have confirmed that the resonance of the fundamental building block of the proposed sensor can be precisely predicted by the established theory based on the Fabry-Pérot cavities and the height-dependent dispersion relation of GPs. It was verified that the established theory 
a

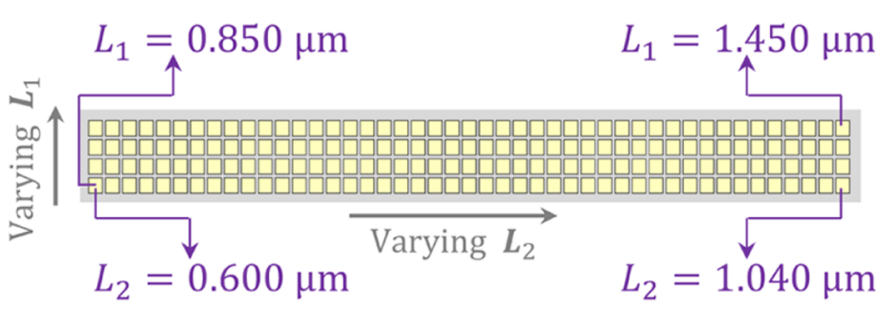

f $\quad$ DMMP, $\lambda_{0}=11.2 \mu \mathrm{m}$

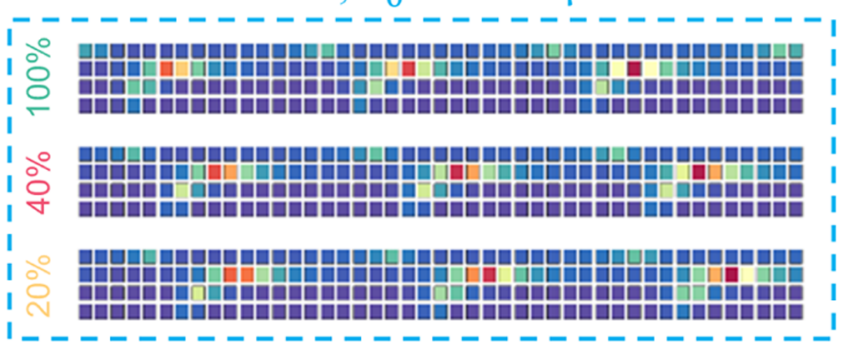

e Three Concentrations of DMMP

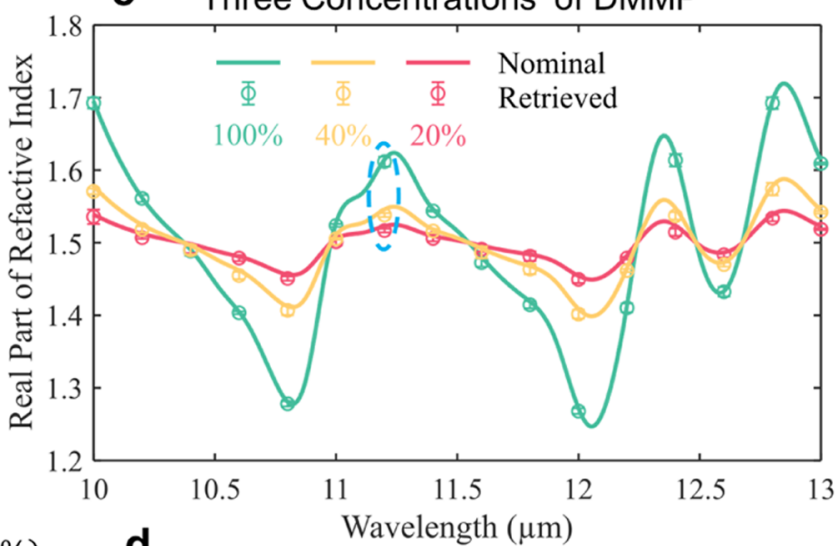

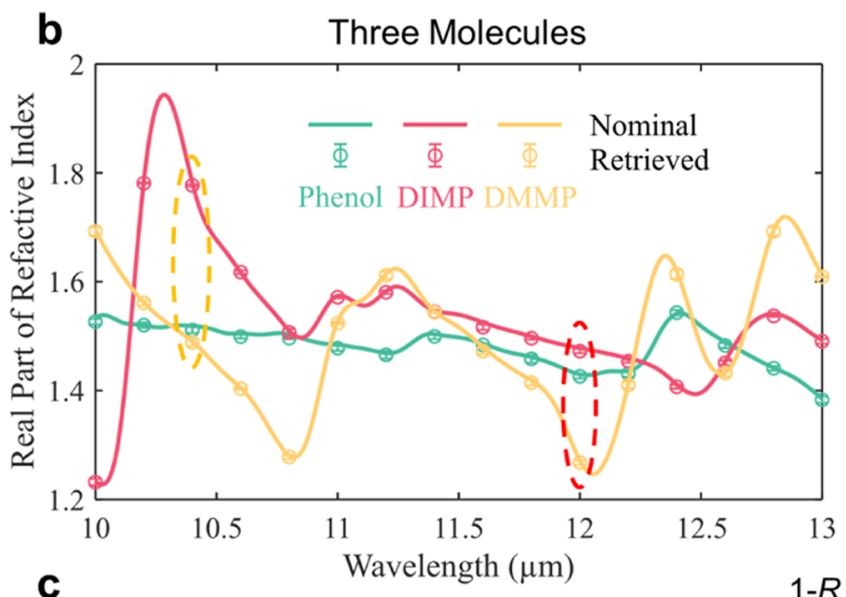

$R(\%)$

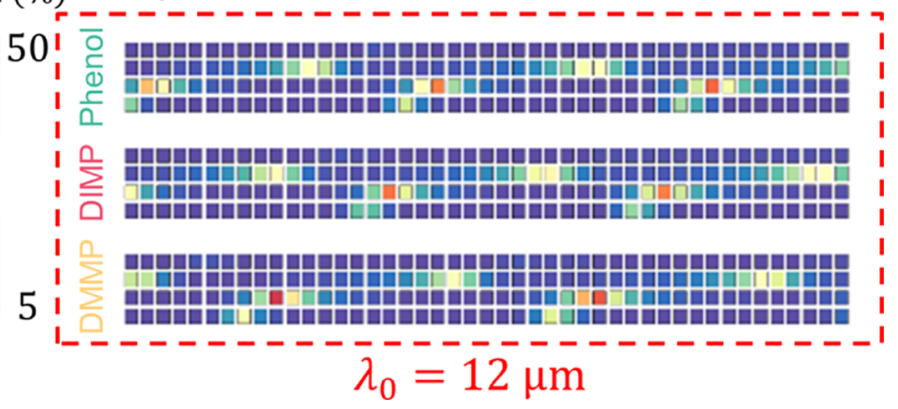

Figure 4. Molecular refractive index retrieval and spatial absorption mapping for chemical identification and concentration analysis. (a) Twodimensional array of suspended graphene plasmon nanocavities with varying trench length and ridge length. The trench length in the unit cell varies linearly between 0.850 and $1.450 \mu \mathrm{m}$ in four steps in the vertical direction. The ridge length varies linearly between 0.600 and $1.040 \mu \mathrm{m}$ in 45 steps in the horizontal direction. (b) Nominal and retrieved refractive index of three different molecules. Absorption barcodes of the pixelated sensor at $\lambda_{0}=10.4 \mu \mathrm{m}$ (c) and $\lambda_{0}=12 \mu \mathrm{m}$ (d) for three molecules. (e) Nominal and retrieved refractive index of three different concentrations. (f) Absorption bar-codes of the pixelated sensor at $\lambda_{0}=11.2 \mu \mathrm{m}$ for three concentrations of DMMP.

remained valid when we changed the incident wavelength and the parameters of this configuration, such as the gap thickness, the cavity height, the Fermi energy of the graphene layer, and the materials filled in the gap and the trench. ${ }^{36,38}$ In the following, we will use the deep subwavelength Fabry-Pérot cavities formed in the system for molecular refractive index measurement.

To illustrate the working principle of the refractive index measurement using our tiny Fabry-Pérot cavity, we first consider an ideal Fabry-Pérot cavity. Depending on the length of the cavity, a greater or smaller number of interference fringes inside the cavity are observed. According to the conventional Fabry-Pérot equation, the real part of the refractive index of the cavity core can be expressed as $n_{\mathrm{c}}=\lambda_{0} /$ $(2 \Delta l)$, where $\Delta l$ is the difference of the cavity lengths for the adjacent modes. In the system studied here, the GPs form deep subwavelength Fabry-Pérot cavities. Consequently, $n_{\mathrm{c}}$ in the above equation should be $\mathcal{R}\left\{N_{\text {eff }}^{h}\right\}$.
To observe the resonances, we propose measuring the reflection (or absorption) pattern of a two-dimensional array of suspended graphene plasmon nanocavities, with two variables of the configuration in the horizontal and vertical directions (Figure 2). Although there are several choices for these two variables, we choose $L_{1}$ and $L_{2}$ here (Figure $2 \mathrm{e}, \mathrm{f}$ ), respectively, due to the convenience of the fabrication. The key points of the proposed technique are to extract the effective wavelength of GPs from the measured absorption pattern based on the Fabry-Pérot equation and then calculate the refractive index of the analyte using the dispersion relation. Although there are several different approaches to realize this technique, in this demonstration (Figure $3 b$ ), the value of $\lambda_{\mathrm{GP}}^{h_{2}}$ is extracted by calculating the separation of adjacent resonance peak positions via

$$
\lambda_{\mathrm{GP}}^{h_{2}}=2 \Delta L_{2} /\left(m_{\mathrm{b}}^{h_{2}}-m_{\mathrm{a}}^{h_{2}}\right)=\lambda_{0} / \mathcal{R}\left\{N_{\mathrm{eff}}^{h_{2}}\right\}
$$

where $m_{\mathrm{b}}^{h_{2}}$ and $m_{\mathrm{a}}^{h_{2}}$ are the orders of the modes excited, respectively, and $\Delta L_{2}$ is the difference of the ridge lengths for 


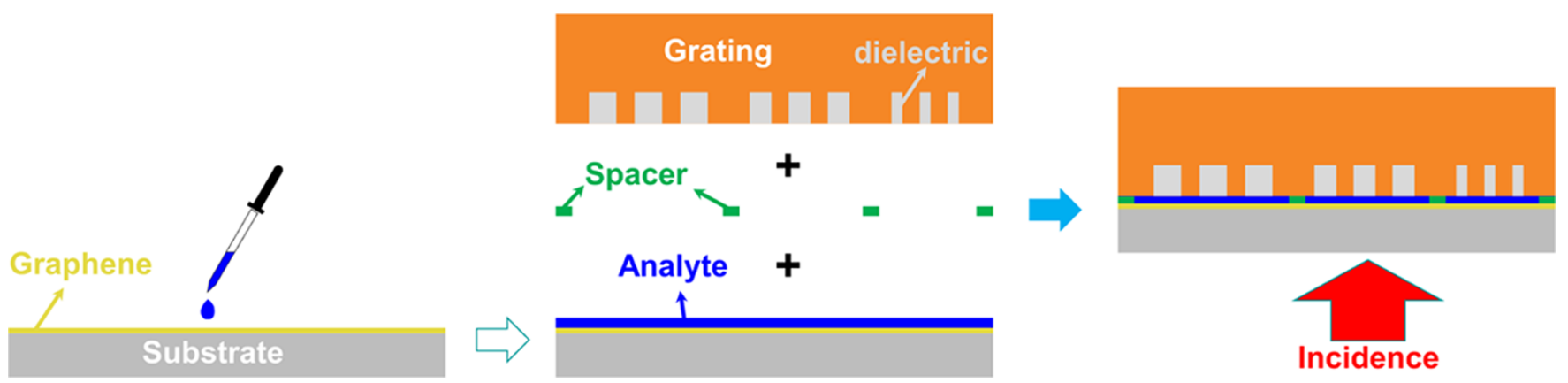

Figure 5. Schematic of a realistic configuration of a tunable digital sensor with suspended graphene plasmon nanocavities. The graphene is transferred on a substrate which is transparent at the interested frequencies. The cavity in the metallic grating is filled with a dielectric medium. The liquid analyte covers the substrate. The grating is transferred on the substrate. The gap with a desired thickness is achieved by varying a spacer between the substrate and the grating. The light is coming from the bottom substrate side.

those two modes. Although the absolute values of $m_{\mathrm{a}}^{h_{2}}$ and $m_{\mathrm{b}}^{h_{2}}$ are usually difficult to be measured in experiments, their difference would be easily obtained by counting the number of resonance peaks. This makes the proposed method a calibration-free method. It is also worth noting that the "Sigmoid function" shape of the strong absorption pattern makes the resonances straightforward to find in the absorption pattern because even when the Fabry-Pérot resonance is not fully satisfied in each cavity, the modes can still be excited and thus be observable. The separation of the adjacent resonance peaks observed for different trench lengths is the same (Figure $3 \mathrm{~b}$ ), indicating that the nonzero phase shift do not affect the proposed technique for the refractive index determination. Such a phenomenon is particularly attractive in the design of the sensor because it allows robust observation of the resonance modes and the adjacent resonance peaks in the captured image. Then, the real part of the refractive index of the analyte could be numerically calculated based on the dispersion relation of GPs. Consequently, the type, even the concentration, of the molecule to be analyzed can be differentiated based on the retrieved refractive index.

We demonstrate the performance of the proposed sensor by showing its capability of measuring the real part of the refractive index for three different molecules. Without any loss of generality, we select the incident wavelength ranging from 10 to $13 \mu \mathrm{m}$. As shown in Figure 4, a two-dimensional array of suspended graphene plasmon nanocavities with varying trench lengths and ridge lengths is used. It should be further noted that a flow cell is placed in the gap over the ridge and on top of the trench to circulate liquid samples and to be reusable (Figure 2c). The flow cell may also serve to support the graphene layer. The thickness of the flow cell is only $5 \mathrm{~nm}$, which means the volume of the analyte required in the measurement is very small. The sensor is used to detect three different molecules, including phenol, ${ }^{39}$ diisopropyl methylphosphonate $^{40}$ (DIMP) and dimethyl methylphosphonate ${ }^{40}$ (DMMP). It should be noted that, in the simulation, only the real part of the reflective index of the analyte is used as a proof of concept. The refractive index here is used to trigger the resonance of the system, and the energy is mainly absorbed by the graphene layer, rather than the analyte itself. This is particularly suitable for the measurement of the refractive index with a negligible imaginary part, in which case the approaches based on characteristic molecular absorption fingerprints would fail. As shown in Figure $4 b$, the retrieved refractive indices of different molecules are in good agreement with the nominal ones, which confirms the validation of the proposed techniques.

Here, we also assess the capability of our sensor for concentration measurement (Figure 4e). For techniques based on the SEIRA concept, when the detected absorption fingerprints of different molecules overlap, it is difficult to differentiate the types of analytes. In addition, for traditional nanophotonic sensors, the signal-to-noise ratio is relatively low. For these reasons, it is very challenging to determine the concentration of molecules using current nanophotonic systems. However, our approach is based on sensing the real part of the refractive index of the analyte. Therefore, this technique would be more suitable for the determination of both the types and concentrations of various molecules. Figure $4 \mathrm{~b}$, e demonstrates that the proposed technique is suitable for refractive index measurement, chemical identification, and concentration analysis. Moreover, the refractive index retrieval at a particular wavelength in this technique relies only on a single measurement, which is an advantage in sensing. Such single measurement detection is beneficial to high-throughput measurement.

Until now, the method for the molecule detection is based on the retrieved refractive indices calculated from the spatial absorption maps. In fact, the barcode-like spatial absorption maps of the two-dimensional array can be used directly for a molecule or concentration sensing, when a database is prepared based on the proposed theory. As shown in Figure 4, different barcode-like spatial absorption maps for different wavelengths, molecules (Figure $4 \mathrm{c}, \mathrm{d}$ ), or concentrations (Figure 4f) are easily observed. Thus, this direct digital imaging technique offers the potential for chemical identification and concentration analysis through a pattern recognition based on a library of multiple molecular barcode signatures.

Additionally, our scheme could provide an ultra-broad spectral measurement band, thanks to the broad band of GPs. When using resonance elements in traditional nanophotonic sensors, the resonance spectrum must be tuned to overlap with the molecular fingerprints. However, the tunability is very limited because it is achieved by varying the geometry or the optical properties (such as the permittivity) of the resonator. Hence, the spectral range measured by the traditional nanophotonic sensors is usually narrow. In contrast, our technique is valid when the plasmons in the graphene layer can be excited efficiently, and thus, its measurable spectral range could cover the mid- and far-IR and terahertz bands by 
carefully designing the two horizontal Fabry-Pérot cavities and the vertical Fabry-Pérot cavities. Finally, a realistic configuration of the sensor is suggested in Figure 5 to produce a flat graphene layer and to precisely control the gap thickness (also see Supporting Information).

\section{CONCLUSIONS}

In summary, we have designed a digital sensor using suspended graphene plasmon nanocavities, which could provide extremely high field enhancement and extraordinary field compression. To do this, we have established the theory of the suspended graphene plasmon nanocavities. In the proposed theory, the Fabry-Pérot equation is used to predict the conditions for the GP resonance in two types of cavities (in the trench and above the ridge), and the dispersion relation of GPs in a general multilayer system is used to calculate the GP wavelength at different values of the cavity height. The excellent agreement between the predictions from this proposed theory and the numerical results confirms the validity of the proposed model. We also have achieved the extremely high field enhancement and extraordinary compression of GPs simultaneously, thanks to the combination of the shallow cavity and the deep cavity in the same configuration. Based on the optical properties of suspended graphene plasmon nanocavities, we have proposed the working principle of a digital sensor that allows the complex refractive index of an analyte to be retrieved. To demonstrate the performance of such a sensor, different molecules and different concentrations of DMMP have been detected. Given the capabilities of the refractive index measurement, the ultra-broadband measurable spectral range, the high sensitivity, and the small volume of the analyte required, the proposed sensor could serve as an ideal platform for applications such as chemical sensing, thermal imaging, heat scavenging, security and materials inspection, and molecule sensing. ${ }^{41-43}$ Furthermore, the tunability of the Fermi energy and geometric parameters of the cavities makes the design of this system extremely versatile. We also expect the investigated system using the combination of the shallow and deep cavities to open up numerous potential applications in photodetection, nonlinear optics, and integrated optics.

\section{ASSOCIATED CONTENT}

\section{SI Supporting Information}

The Supporting Information is available free of charge at https://pubs.acs.org/doi/10.1021/acsanm.0c03100.

Height-dependent dispersion relationship of GPs, simulation details, discussions of the realistic configuration $(\mathrm{PDF})$

\section{AUTHOR INFORMATION}

\section{Corresponding Author}

Xiaofei Xiao - The Blackett Laboratory, Imperial College London, London SW7 2AZ, U.K.; ○ orcid.org/0000-00027053-2833; Email: xiaofei.xiao15@imperial.ac.uk

\section{Authors}

Stefan A. Maier - The Blackett Laboratory, Imperial College London, London SW7 2AZ, U.K.; Chair in Hybrid Nanosystems, Nanoinstitute München, Faculty of Physics, Ludwig-Maximilians-Universität München, 80539 München, Germany
Vincenzo Giannini - Instituto de Estructura de la Materia (IEM-CSIC), Consejo Superior de Investigaciones Cientificas, 28006 Madrid, Spain; Technology Innovation Institute, Masdar City, Abu Dhabi, United Arab Emirates; (1) orcid.org/0000-0001-8025-4964

Complete contact information is available at: https://pubs.acs.org/10.1021/acsanm.0c03100

\section{Notes}

The authors declare no competing financial interest.

\section{ACKNOWLEDGMENTS}

X.X. is supported by Lee Family Scholars. S.M. acknowledges the Lee-Lucas Chair in Physics. V.G. acknowledges the Spanish Ministerio de Economia y Competitividad for financial support through the grant NANOTOPO (FIS2017-91413-EXP) and also the Ministerio de Ciencia, Innovación y Universidades through the grant MELODIA (PGC2018-095777-B-C21). The authors thank Dr. Daan M. Arroo and Marie Rider for comments on the manuscript.

\section{REFERENCES}

(1) Chalmers, J.; Griffiths, P. Handbook of Vibrational Spectroscopy; Wiley, 2002; Vol. 5.

(2) Mizaikoff, B. Waveguide-enhanced mid-infrared chem/bio sensors. Chem. Soc. Rev. 2013, 42, 8683-8699.

(3) Gómez Rivas, J.; Vecchi, G.; Giannini, V. Surface plasmon polariton-mediated enhancement of the emission of dye molecules on metallic gratings. New J. Phys. 2008, 10, 105007.

(4) Li, R.; Wu, D.; Liu, Y.; Yu, L.; Yu, Z.; Ye, H. Infrared plasmonic refractive index sensor with ultra-high figure of merit based on the optimized all-metal grating. Nanoscale Res. Lett. 2017, 12, 1-6.

(5) Leitis, A.; Tittl, A.; Liu, M.; Lee, B. H.; Gu, M. B.; Kivshar, Y. S.; Altug, H. Angle-multiplexed all-dielectric metasurfaces for broadband molecular fingerprint retrieval. Sci. Adv. 2019, 5, No. eaaw2871.

(6) Tittl, A.; Leitis, A.; Liu, M.; Yesilkoy, F.; Choi, D.-Y.; Neshev, D. N.; Kivshar, Y. S.; Altug, H. Imaging-based molecular barcoding with pixelated dielectric metasurfaces. Science 2018, 360, 1105-1109.

(7) Neubrech, F.; Huck, C.; Weber, K.; Pucci, A.; Giessen, H. Surface-enhanced infrared spectroscopy using resonant nanoantennas. Chem. Rev. 2017, 117, 5110-5145.

(8) Dong, L.; Yang, X.; Zhang, C.; Cerjan, B.; Zhou, L.; Tseng, M. L.; Zhang, Y.; Alabastri, A.; Nordlander, P.; Halas, N. J. Nanogapped $\mathrm{Au}$ antennas for ultrasensitive surface-enhanced infrared absorption spectroscopy. Nano Lett. 2017, 17, 5768-5774.

(9) Singh, P. SPR biosensors: Historical perspectives and current challenges. Sensor. Actuator. B Chem. 2016, 229, 110-130.

(10) Giannini, V.; Zhang, Y.; Forcales, M.; Gómez Rivas, J. Longrange surface polaritons in ultra-thin films of silicon. Opt. Express 2008, 16, 19674-19685.

(11) Giannini, V.; Fernández-Domínguez, A. I.; Heck, S. C.; Maier, S. A. Plasmonic nanoantennas: fundamentals and their use in controlling the radiative properties of nanoemitters. Chem. Rev. 2011, 111, 3888-3912.

(12) Biagioni, P.; Huang, J.-S.; Hecht, B. Nanoantennas for visible and infrared radiation. Rep. Prog. Phys. 2012, 75, 024402.

(13) Jahani, S.; Jacob, Z. All-dielectric metamaterials. Nat. Nanotechnol. 2016, 11, 23-36.

(14) Ran, Z. L.; Rao, Y. J.; Liu, W. J.; Liao, X.; Chiang, K. S. Lasermicromachined Fabry-Perot optical fiber tip sensor for highresolution temperature-independent measurement of refractive index. Optic Express 2008, 16, 2252-2263.

(15) Ran, Z.; Rao, Y.; Zhang, J.; Liu, Z.; Xu, B. A miniature fiberoptic refractive-index sensor based on laser-machined Fabry-Perot interferometer tip. J. Lightwave Technol. 2009, 27, 5426-5429. 
(16) Grigorenko, A. N.; Polini, M.; Novoselov, K. S. Graphene plasmonics. Nat. Photon. 2012, 6, 749.

(17) García de Abajo, F. J. Graphene plasmonics: challenges and opportunities. ACS Photonics 2014, 1, 135-152.

(18) Rappoport, T. G.; Epstein, I.; Koppens, F. H. L.; Peres, N. M. R. Understanding the electromagnetic response of graphene/metallic nanostructures hybrids of different dimensionality. ACS Photonics 2020, 7, 2302-2308.

(19) Wang, R.; Ren, X.-G.; Yan, Z.; Jiang, L.-J.; Wei, E.; Shan, G.-C. Graphene based functional devices: A short review. Front. Phys. 2019, $14,13603$.

(20) Low, T.; Avouris, P. Graphene plasmonics for terahertz to midinfrared applications. ACS Nano 2014, 8, 1086-1101.

(21) Huang, L.; Hu, G.; Deng, C.; Zhu, Y.; Yun, B.; Zhang, R.; Cui, Y. Realization of mid-infrared broadband absorption in monolayer graphene based on strong coupling between graphene nanoribbons and metal tapered grooves. Optic Express 2018, 26, 29192-29202.

(22) Fei, Z.; Rodin, A. S.; Andreev, G. O.; Bao, W.; McLeod, A. S.; Wagner, M.; Zhang, L. M.; Zhao, Z.; Thiemens, M.; Dominguez, G.; Fogler, M. M.; Neto, A. H. C.; Lau, C. N.; Keilmann, F.; Basov, D. N. Gate-tuning of graphene plasmons revealed by infrared nano-imaging. Nature 2012, 487, 82-85.

(23) Chen, J.; Badioli, M.; Alonso-González, P.; Thongrattanasiri, S.; Huth, F.; Osmond, J.; Spasenović, M.; Centeno, A.; Pesquera, A.; Godignon, P.; Zurutuza Elorza, A.; Camara, N.; de Abajo, F. J. G.; Hillenbrand, R.; Koppens, F. H. L. Optical nano-imaging of gatetunable graphene plasmons. Nature 2012, 487, 77-81.

(24) Rufangura, P.; Folland, T. G.; Agrawal, A.; Caldwell, J. D.; Iacopi, F. Towards low- loss on-chip nanophotonics with coupled graphene and silicon carbide: a review. J. Mater. Phys. 2020, 3, 032005 .

(25) Koppens, F. H. L.; Mueller, T.; Avouris, P.; Ferrari, A. C.; Vitiello, M. S.; Polini, M. Photodetectors based on graphene, other two-dimensional materials and hybrid systems. Nat. Nanotechnol. 2014, 9, 780-793.

(26) Shautsova, V.; Sidiropoulos, T.; Xiao, X.; Güsken, N. A.; Black, N. C.; Gilbertson, A. M.; Giannini, V.; Maier, S. A.; Cohen, L. F.; Oulton, R. F. Plasmon induced thermoelectric effect in graphene. Nat. Commun. 2018, 9, 5190.

(27) Konstantatos, G. Current status and technological prospect of photodetectors based on two-dimensional materials. Nat. Commun. 2018, 9, 5266.

(28) Nguyen-Huu, N.; Pištora, J.; Cada, M.; Nguyen-Thoi, T.; Ma, Y.; Yasumoto, K.; Rahman, B. A.; Wu, Q.; Ma, Y.; Ngo, Q. H.; Jie, L.; Maeda, H. Ultra-Wide Spectral Bandwidth and Enhanced Absorption in a Metallic Compound Grating Covered by Graphene Monolayer. IEEE J. Sel. Top. Quantum Electron. 2020, 27, 1-8.

(29) Huang, L.; Liu, J.; Deng, H.; Wu, S. Phonon-Like Plasmonic Resonances in a Finite Number of Graphene Nanoribbons. Adv. Opt. Mater. 2018, 6, 1701378 .

(30) Ogawa, S.; Fukushima, S.; Shimatani, M. Graphene Plasmonics in Sensor Applications: A Review. Sensors 2020, 20, 3563.

(31) Epstein, I.; Alcaraz, D.; Huang, Z.; Pusapati, V.-V.; Hugonin, J.P.; Kumar, A.; Deputy, X. M.; Khodkov, T.; Rappoport, T. G.; Hong, J.-Y.; Peres, N. M. R.; Kong, J.; Smith, D. R.; Koppens, F. H. L. Farfield excitation of single graphene plasmon cavities with ultracompressed mode volumes. Science 2020, 368, 1219-1223.

(32) Rodrigo, D.; Limaj, O.; Janner, D.; Etezadi, D.; Garcia de Abajo, F. J.; Pruneri, V.; Altug, H. Mid-infrared plasmonic biosensing with graphene. Science 2015, 349, 165-168.

(33) Xiao, S.; Zhu, X.; Li, B.-H.; Mortensen, N. A. Grapheneplasmon polaritons: From fundamental properties to potential applications. Front. Phys. 2016, 11, 117801.

(34) Wenger, T.; Viola, G.; Kinaret, J.; Fogelström, M.; Tassin, P. High-sensitivity plasmonic refractive index sensing using graphene. 2D Materials 2017, 4, 025103.

(35) Yi, Z.; Liang, C.; Chen, X.; Zhou, Z.; Tang, Y.; Ye, X.; Yi, Y.; Wang, J.; Wu, P. Dual-band plasmonic perfect absorber based on graphene metamaterials for refractive index sensing application. Micromachines 2019, 10, 443.

(36) Xiao, X.; Li, X.; Caldwell, J. D.; Maier, S. A.; Giannini, V. Theoretical analysis of graphene plasmon cavities. Appl. Mater. Today 2018, 12, 283-293.

(37) Li, K.; Fitzgerald, J. M.; Xiao, X.; Caldwell, J. D.; Zhang, C.; Maier, S. A.; Li, X.; Giannini, V. Graphene Plasmon Cavities Made with Silicon Carbide. ACS Omega 2017, 2, 3640-3646.

(38) Xiao, X. Graphene plasmon cavities. Ph.D. Thesis, Imperial College London, 2019.

(39) Francescato, Y.; Giannini, V.; Yang, J.; Huang, M.; Maier, S. A. Graphene sandwiches as a platform for broadband molecular spectroscopy. ACS Photonics 2014, 1, 437-443.

(40) Querry, M. R. Optical Constants of Minerals and other Materials from the Millimeter to the Ultraviolet; Chemical Research, Development \& Engineering Center, US Army Armament, 1987.

(41) Germain, M. E.; Knapp, M. J. Optical explosives detection: from color changes to fluorescence turn-on. Chem. Soc. Rev. 2009, 38, $2543-2555$.

(42) Long, F.; Zhu, A.; Shi, H. Recent advances in optical biosensors for environmental monitoring and early warning. Sensors 2013, 13, $13928-13948$.

(43) Fan, X.; White, I. M.; Shopova, S. I.; Zhu, H.; Suter, J. D.; Sun, Y. Sensitive optical biosensors for unlabeled targets: A review. Anal. Chim. Acta 2008, 620, 8-26. 